\title{
ETNOBOTÁNICA, FITOQUÍMICA Y FARMACOLOGÍA DE ESPECIES DEL GÉNERO Baccharis (ASTERACEAS) UTILIZADAS COMO PLANTAS MEDICINALES EN EL DEPARTAMENTO DE AYACUCHO
}

\section{Ethnobotany, phytochermistry and pharmacology of medicinal plants belonging to the genus Baccharis (Asteraceas) used at Ayacucho city}

\author{
Enrique Aguilar ${ }^{1}$, Brita Anaya ${ }^{2}$, José Alarcón ${ }^{2}$, Aldo Tinco ${ }^{2}$
}

\section{RESUMEN}

El objetivo fue estudiar especies del género Baccharis desde el punto de vista etnobotánico, químico y farmacológico utilizadas como plantas medicinales en el departamento de Ayacucho. Tres especies del género Baccharis fueron colectadas en el distrito de Quinua y en el Valle de Muyurina (provincia de Huamanga, Ayacucho), durante los meses de abril a junio del 2006. El estudio etnobotánico se realizó según metodología de Cáceres (1996) y el fitoquímico según Miranda y Cuellar (2000), la actividad antiinflamatoria mediante el método de edema plantar inducido por prostaglandina E1, la actividad antioxidante por la actividad secuestradora del 1,1-difenil-picril-hidrazilo (DPPH) y el antimicrobiano por el método de placa excavada. Las especies fueron identificadas como: $B$. salicifolia utilizada como analgésico, antiinflamatorio y antirreumático; $B$. genistelloides como antiinflamatorio y en problemas hepáticos; y de B. glutinosa no se reportan usos conocidos. Se aislaron flavonoides de tipo flavona y flavanona de las hojas de las tres especies en estudio. $B$. salicifolia demostró tener mejor actividad antiinflamatoria $(\mathrm{p}<0.05)$ y antioxidante $(\mathrm{p}<0.05)$ que $B$. genistelloides y $B$. glutinosa; mientras que $B$. glutinosa presentó actividad antimicrobiana frente a Salmonella sp. y Shigella sonnei. Se identificó tres especies: $B$. genistelloides, $B$. glutinosa y $B$. salicifolia; y se realizó el estudio etnobotánico que reportan su uso tradicional. Los flavonoides presentes en las especies son de tipo flavona y flavanona. $B$. salicifolia fue mejor antiinflamatorio y antioxidante; B. glutinosa mejor antimicrobiano.

Palabras clave: Baccharis, etnobotánica, fitoquímica, farmacología.

\section{SUMMARY}

Species from Baccharis genus were studied fo the viewpoint ethnobotanical, chemical and pharmacological which are used as medicinal piants at de Ayacucho department. Three species from Baccharis genus were collected from the Quinua district and the Muyurina Valley (Huamanga province, Ayacucho), during april to june months of 2006. The ethnobotanical study was realized using the Caceres's methodologie(1996) and the phytochemical study in according to Miranda and Cuellar (2000), prostaglandine E1-induced rat paw oedema has been used as an anti-inflammatory model, the antioxidant activity was studied using the DPPH scavenger and the antimicrobial activity with the excavated plate model. The species were identified as B. salicifolia used as analgesic, anti-inflammatory and anti-rheumatic; $B$. genistelloides as anti-inflammatory and hepatic problems and of the B. glutinosa wasn't reported any know use. It were isolated flavonoids of type flavone and flavanone of the leaves from the Baccharis. B. salicifolia has demonstrated have the best anti-inflammatory activity $(\mathrm{p}<0.05)$ and antioxidant $(\mathrm{p}<0.05)$; while $B$. glutinosa has presented antimicrobial activity againts Salmonella $s p$. and Shigella sonnei. Three species of Baccharis genus were identified as B. genistelloides, B. glutinosa y B. salicifolia; and the ethnobotanical study was performed reporting the traditional uses. The flavonoids present are the flavone and flavonone type. B. Salicifolia was the best anti-inflammatory and antioxidant; $B$. glutinosa was the best antimicrobial.

Key words: Baccharis, ethnobotanical, phytochemistry, pharmacology.

\footnotetext{
1 Instituto de Recursos Vegetales y Terapéuticos, Facultad de Farmacia y Bioquímica, Universidad Nacional Mayor de San Marcos.

2 Universidad Nacional de San Cristóbal de Huamanga. pbonillar@unmsm.edu.pe
} 


\section{INTRODUCCIÓN}

La familia Asteracea es uno de los grupos sistemáticos más numerosos dentro de las Angiospermas, que comprende 1100 géneros y cerca de 25000 especies. (De la Cruz y Romero, 1997) (De la Cruz y Romero, 1999).

El género Baccharis (familia Asteraceae) está representada por más de 500 especies, distribuidas principalmente en el Brasil, Argentina, Colombia, Chile, México, ocupando las regiones más elevadas. Una alta densidad de especies en el Brasil y en los Andes, indica que unas de esas áreas son áreas probables de origen de esos géneros. En el Brasil, están descritas 120 especies del género Baccharis, con una mayor parte de ellas localizadas en la región sudeste del país. Se estima en 100 especies en Argentina, 28 en México y cerca de 40 en Colombia, constituyendo uno de los dos grupos de plantas más importantes en este país, de las cuales el $38 \%$ son endémicas (Gonzaga y col, 2005)

Existe un interés muy grande por estudiar a éste género por su amplio uso en la medicina tradicional, debido a su contenido fitoquímico de flavonoides, diterpenos y triterpenos. (Gonzaga y col, 2005)

En Ayacucho, el genero Bacharis está constituido por muchas especies utilizadas en la medicina tradicional, que necesitan ser estudiadas desde el punto de vista etnobotánico, químico y farmacológico, como una contribución para su aprovechamiento racional por parte de nuestra población.

Por eso se plantea el presente trabajo de investigación para identificar especies de interés del género Baccharis $y$ hacer estudios para determinar los compuestos quîmicos utilizando el tamizaje fitoquímico, pruebas cromatográficas y espectrales para determinar su naturaleza química; y ensayos farmacológicas para determinar la actividad antiinflamatoria, antioxidante y antimicrobiana.

\section{MATERIALES Y MÉTODOS}

\section{Recolección de muestras}

Se colectaron tres especies del género Baccharis en el Valle de Muyurina y en el distrito de Quinua, provincia de Huamanga durante los meses de abril a junio del 2006, de plantas en estado de floración. Las muestras fueron las hojas, siendo trasladadas al Laboratorio de Farmacia de la Facultad de Ciencias Biológicas de la UNSCH.

\section{Identificación de las especies}

La identificación de las especies colectadas fue realizada por el $\mathrm{Mg}$. Jesús de la Cruz Arango, Profesor Principal del Laboratorio de Botánica de la UNSCH.

\section{Estudio etnobotánico}

Se realizó mediante una encuesta etnobotánica a las especies previamente identificadas, a los pobladores de la zona para conocer sus propiedades medicinales atribuidas, según la metodología propuesta por Cáceres (1996).

\section{Estudio fitoquímico}

Preparación de la muestra. Las muestras fueron desecadas a la sombra y sometidas a molienda en un mortero de porcelana hasta obtener un polvo fino, siendo almacenados en frascos de color ámbar para su conservación y posterior tratamiento.

Obtención de los extractos. Las muestras fueron maceradas con etanol al $80 \%$, realizándose un proceso de doble extracción, obteniéndose los extractos hidroalcohólicos de cada una de las muestras. Estos extractos fueron concentrados en un evaporador rotatorio hasta consistencia siruposa, siendo conservados en refrigeración.

Tamizaje fitoquímico. Para el tamizaje fitoquímico se siguió la metodología propuesta por Miranda y Cuéllar (2000), realizando pruebas de identificación para los metabolitos secundarios presentes en cada uno de los extractos.

Fraccionamiento del extracto etanólico. El extracto etanólico fue fraccionado con solventes de polaridad creciente, obteniéndose fracciones étereas, clorofórmicas, de acetato de etilo y acuosas. En cada de una de ellas se realizaron pruebas cromatográficas. (Lock de Ugaz, 1994).

Pruebas cromatográficas. Se realizaron pruebas cromatográficas, utilizando como sistema de solventes la mezcla de Cloroformo: Metanol en las proporciones de $(6: 1),(9: 1),(12: 1),(15: 1)$ y $(18: 1)$, siendo revelados con Luz UV de $366 \mathrm{~nm}$. Se realizaron cromatografías preparativas, utilizando como solvente Cloroformo: Metanol (12:1), obteniéndose fracciones que fueron recuperadas en metanol y leídas en el Espectrofotometro UV Genesys 6, para obtener su curva espectral, y determinar la naturaleza del compuesto aislado utilizando las Tablas de Mabry y col. (1970). 


\section{Ensayos farmacológicos}

\section{Actividad antiinflamatoria}

La actividad antiinflamatoria se determinó utilizando la metodología de inflamación por edema plantar inducido por Prostaglandina E1 y midiendo el volumen de inflamación en el pletisnometro (CYTED, 1995).

\section{Actividad antioxidante}

Actividad secuestradora del radical 1,1-difenil-2picrilhidrazilo. (Suárez, 2003).

Se realizó utilizando la técnica del 1,1-difenil-picrilhidrazilo (DPPH), el cual es un radical libre estable, el mismo que presenta una coloración púrpura con absorbancia a $517 \mathrm{~nm}$. El DPPH se preparó en metanol a una concentración de $20 \mathrm{mg} / \mathrm{L}$ y se tuvo en cuenta el siguiente procedimiento:

\begin{tabular}{|l|c|c|c|c|}
\hline & Blanco & Control & $\begin{array}{c}\text { Blanco de } \\
\text { Muestra }\end{array}$ & Muestra \\
\hline Agua destilada & 1,00 & 0,75 & - & -- \\
Metanol & 2,00 & -- & 1,50 & -- \\
Muestra & -- & -- & 0,75 & 0,75 \\
DPPH & -- & 1,50 & - & 1,50 \\
\hline
\end{tabular}

Mezclar y dejar en reposo a temperatura ambiente durante 5 minutos y leer a $517 \mathrm{~nm}$. La muestra se preparó a las concentraciones de $300 \mu \mathrm{g} / \mathrm{ml}, 150$ $\mu \mathrm{g} / \mathrm{ml}$ y $30 \mu \mathrm{g} / \mathrm{ml}$, las mismas equivalen al $100 \%$, $50 \%$ y $10 \%$ de la actividad secuestradora de radicales libres respectivamente (Suárez, 2006).

Cálculos:

$\%$ Actividad antioxidante $=1-\frac{\left(A_{2}-A_{3}\right)}{A_{1}} \times 100$

Donde:

$A_{1}$ : Absorbancia del patrón de referencia

$\mathrm{A}_{2}$ : Absorbancia de la muestra

$\mathrm{A}_{3}$ : Absorbancia del blanco de la muestra.

\section{Actividad antimicrobiana}

Se utilizó el método de dilución en placa excavada, haciendo diluciones al $1 \%, 0.5 \%$ y $0.25 \%$ en agua destilada, ensayándose en las siguientes bacterias: Shigella sonnei, Salmonella sp., Staphylococcus au- reus, observándose la presencia de halos de inhibición después de 24 horas de incubación. (Cáceres, 1996).

\section{Análisis de datos}

Los resultados son presentados en forma de tablas y gráficos. Cuando se evalúan más de tres muestras estos son sometidos a un Análisis de Varianza y la prueba complementaria de comparación de medias múltiple con un nivel de confianza de $95 \%$. Cuando se evalúan dos factores se utilizó un análisis factorial simple.

\section{RESULTADOS}

\section{Identificación de las especies}

Las especies colectadas fueron identificadas botánicamente por el Mg. Jesús de la Cruz, de la siguiente manera:

- Baccharis glutinosa

- Baccharis genistelloides

- Baccharis salicifolia

\section{Estudio etnobotánico:}

La especie Baccharis salicifolia es conocido como: chilca, chilca blanca, chilca, ckechua chilca, chillca, chilco, yana chilca, chilca negra y es utilizado como Se utiliza como antiinflamatorio, analgésico, antiespasmódico en forma de infusión. También como cicatrizante, vulnerario y contra las fracturas en forma de emplastos.

El agua de cocimiento de ramas y hojas se bebe para curar o aliviar los dolores reumáticos.

Baccharis genistelloides es conocido como: callua-callua, cuchu-cuchu, ischu tullma, karkeja, kuchu-kuchu, quinsa cuchu, taya, carqueja. Se utiliza como antirreumática, para curar el mal de hígado como desinflamante y colagogo. Para curar dolores reumáticos se hace frotaciones con hojas trituradas; para problemas del hígado se toma cocimientos de hojas y ramas dos veces al día.

Tinte, las hojas se usan para teñir de color amarillo o verde.

Mientras que a Baccharis glutinosa se le confunde y se le reportan los mismos usos que $B$. salicifolia. 


\section{Tamizaje Fitoquímico}

Tabla 1. Tamizaje fitoquímico de los extractos hidroalcohólicos de Baccharis salicifolia, Baccharis genistelloides y Baccharis glutinosa. Ayacucho-2006.

\begin{tabular}{|c|c|c|c|}
\hline $\begin{array}{ll}\text { Metabolitos } & \text { Especie } \\
\text { Secundarios }\end{array}$ & B. glutinosa & B. salicifolia & B. genistelloides \\
\hline Flavonoides & + & + & ++ \\
\hline Taninos y/o compuestos & $H$ & ++ & +++ \\
\hline Fenólicos & & & \\
\hline Triterpenos y Esteroides & $H+$ & ++ & ++ \\
\hline Catequinas & $H$ & +4 & + \\
\hline Sustancias reductoras & ++ & + & +++ \\
\hline Lactonas y Cumarinas & +1 & ++ & + \\
\hline Quinonas & -- & - & + \\
\hline Azúcares reductores & ++ & + & ++ \\
\hline Alcaloides & - & - & - \\
\hline$"$ & -- & - & - \\
\hline “ & - & - & - \\
\hline “ & -- & - & - \\
\hline
\end{tabular}

$(+++)$ Abundante; $(+)$ bastante; $(+)$ poco; $(--)$ ausencia

Tabla 2. Fracciones aisladas del extracto hidroalcohólico de las hojas de Baccharis genistelloides con sus pruebas cualitativas y espectrales de identificación. Ayacucho -2006 .

\begin{tabular}{|c|c|c|c|c|}
\hline Fracciones & $\begin{array}{c}\text { Prueba de } \\
\text { Shinoda }\end{array}$ & $\mathrm{NaOH} \mathrm{10 \%}$ & $\mathrm{FeCl}_{3} \mathbf{1 \%}$ & $\lambda_{\text {max }}^{\mathrm{MeOH}}$ \\
\hline 1 & ++ & +++ & +++ & $241,300,328$ \\
2 & + & + & + & 286,328 \\
3 & + & + & + & 280 \\
4 & + & + & + & $225,270,318$ \\
5 & + & + & + & $225,268,323$ \\
\hline
\end{tabular}

Tabla 3. Fracciones aisladas del extracto hidroalcohólico de las hojas de Baccharis glutinosas con sus pruebas cualitativas y espectrales de identificación. Ayacucho -2006.

\begin{tabular}{|c|c|c|c|c|}
\hline Fracciones & $\begin{array}{c}\text { Prueba de } \\
\text { Shinoda }\end{array}$ & $\mathrm{NaOH} 10 \%$ & $\mathrm{FeCl}_{3} \mathbf{1} \%$ & $\lambda_{\text {max }}^{\mathrm{MeOH}}$ \\
\hline 1 & ++ & ++ & ++ & $241,300,328$ \\
2 & + & + & + & $268,296 \mathrm{sh}, 333$ \\
3 & + & + & + & $281,364 \mathrm{sh}$ \\
4 & + & + & + & 271,336 \\
5 & + & + & + & 268,323 \\
\hline
\end{tabular}

Tabla 4. Fracciones aisladas del extracto hidroalcohólico de las hojas de Baccharis salicifolia con sus pruebas cualitativas y espectrales de identificación. Ayacucho - 2006.

\begin{tabular}{|c|c|c|c|c|}
\hline Fracciones & $\begin{array}{c}\text { Prueba de } \\
\text { Shinoda }\end{array}$ & $\mathrm{NaOH} \mathrm{10 \%}$ & $\mathrm{FeCl}_{3} 1 \%$ & $\lambda_{\max }^{\mathrm{MeOH}}$ \\
\hline 1 & +++ & ++ & ++ & $250,294,307 \mathrm{sh}$ \\
2 & + & + & + & 242,340 \\
3 & + & + & + & 290 \\
4 & ++ & + & + & $253,314 \mathrm{sh}, 323$ \\
5 & + & + & + & $289,325 \mathrm{sh}$ \\
\hline
\end{tabular}




\section{Ensayos farmacológicos:}

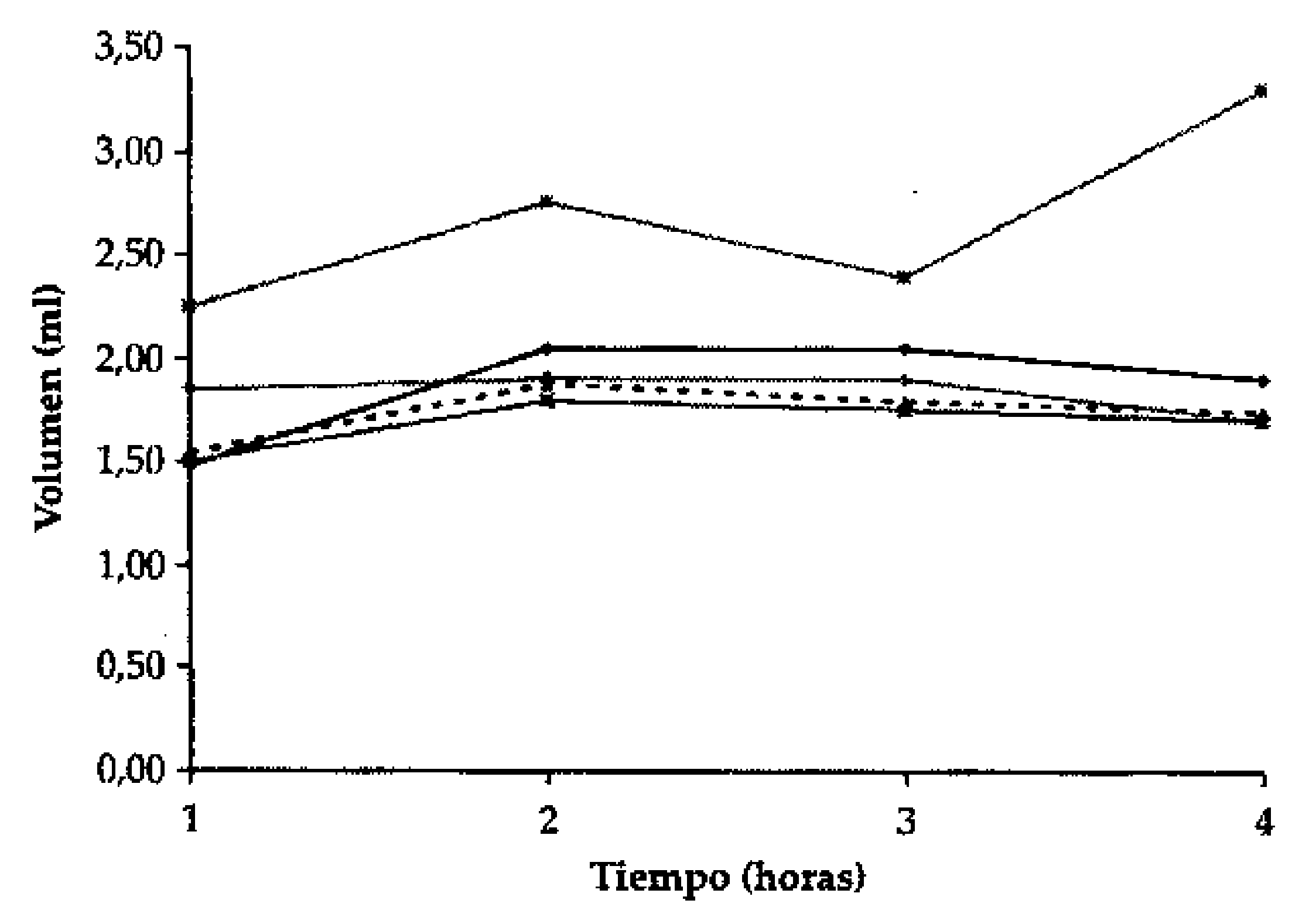

\begin{tabular}{|lc|}
\hline Blanco - - PGE1 & - - B. genistelloides $250 \mathrm{mg} / \mathrm{kg}$ \\
- B. glutinosa $250 \mathrm{mg} / \mathrm{kg}$ & $\rightarrow-$ B. silicifolia $250 \mathrm{mg} / \mathrm{kg}$ \\
\hline
\end{tabular}

Figura 1. Variación del volumen en función del tiempo por efecto antiinflamatorio de los extractos hidroalcohólicos de Baccharis genistelloides, Baccharis glutinosa y Baccharis salicifolia. Ayacucho, 2006.

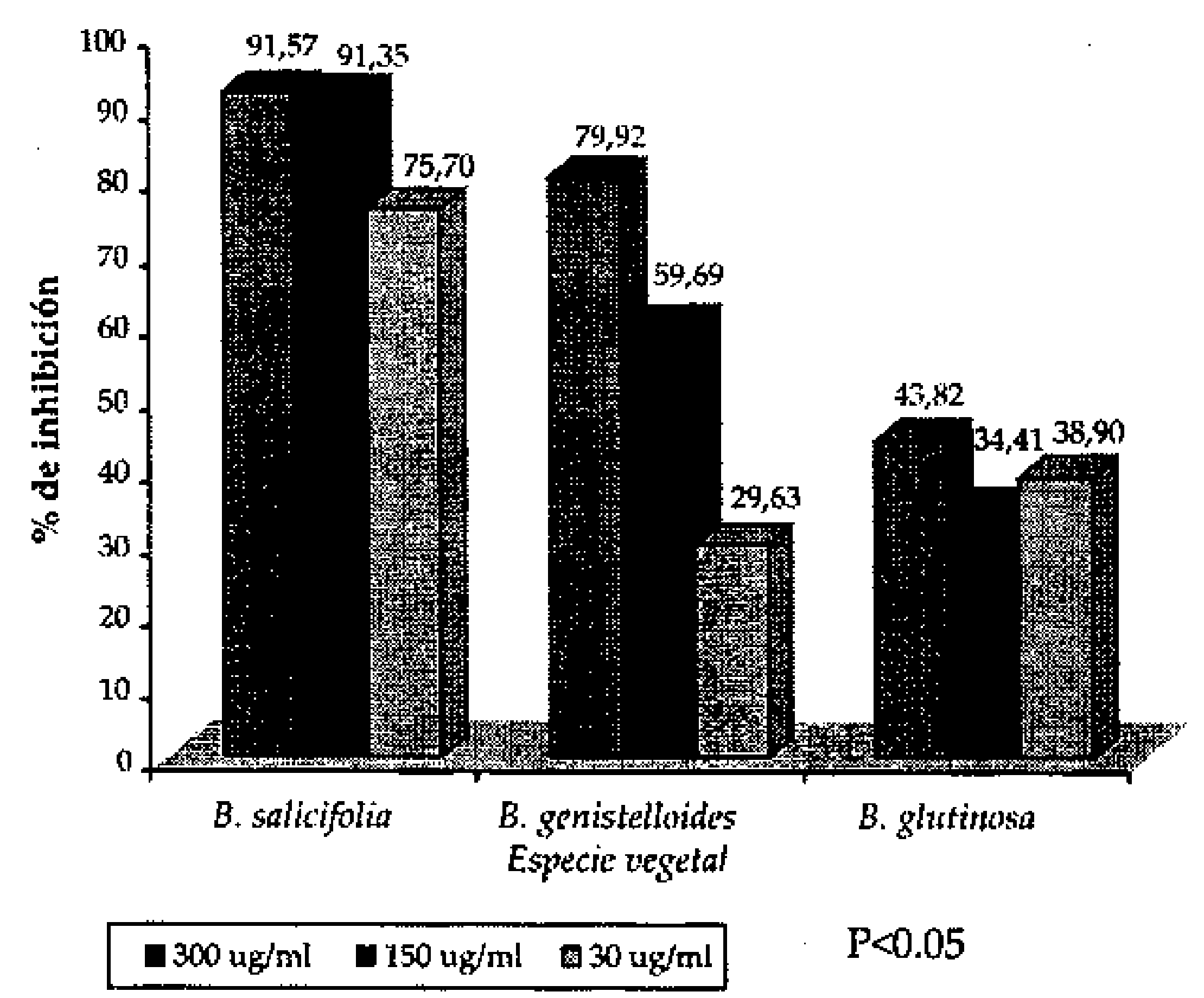

Figura 2. Porcentaje de inhibición del DPPH de los extractos hidroalcoholicos de Baccharis salicifolia, Baccharis genistelloides y Baccharis glutinosa. Ayacucho, 2006.

Tabla 5. Actividad antibacteriana de los extractos hidroalcohólicos de Baccharis salicifolia, Baccharis genistelloides y Baccharis glutinosa.

\begin{tabular}{|l|c|c|c|}
\hline Bacteria & B.glutinosa & B. salicifolia & B. genistelloides \\
\hline Shigella sonnei & Sí & No & No \\
Salmonella $s p$. & Sí & No & No \\
Staphylococcus aureus & No & No & No \\
\hline
\end{tabular}

\section{DISCUSIÓN}

Para el presente trabajo se seleccionó a tres especies del género Baccharis que tienen bastante ocurrencia en lugares cercanos a la ciudad de Ayacucho: Baccharis salicifolia, Baccharis genistelloides y Baccharis glutinosa. B. salicifolia y B. genistelloides son especies que se comercializan ampliamente como plantas medicinales; mientras que a $B$. glutinosa, es una especies que se le confunde como $B$. salicifolia y se le asigna los mismos usos.

Las especies fueron identificadas por el $\mathrm{Mg}$. De la Cruz, del Laboratorio de Botánica de la Universidad Nacional San Cristóbal de Huamanga, quien certificó cada una de las especies y señaló que $B a c-$ charis glutinosa es una especie diferente que $B$. salicifolia.

La información etnobotánica obtenida en la población ayacuchana, en donde $B$. salicifolia es ampliamente utilizada por nuestra población como analgésico, antiinflamatorio y antirreumático, es decir que corresponde a las propiedades de los fármacos llamados Antiinflamatorios No estereoideos (AINES) (Brack, 1999) (Palacios, 1997) (Tovar, 2001). Arones (1998) determinó su actividad antiinflamatoria experimentalmente. Mientras que $B$. genistelloides tiene un uso mixto entre nuestra población, es decir que se utiliza como antiinflamatorio y para problemas hepáticos, sin embargo, el uso para problemas del hígado es el más difundido. Castro (2000), realizó un estudio para determinar la actividad gastroprotectora de esta especie. $B$. glutinosa es confundida como $B$. salicifolia y por lo tanto nuestra población la utiliza indistintamente como esa especie.

En la tabla 1, se observa el tamizaje fitoquímico de las tres especies, destacando la presencia de flavonoides, terpenos y esteroides, sustancias reductoras, lactonas y cumarinas. Podemos afirmar que químicamente el género Baccharis contiene los mismos compuestos, pero seguramente difieren en la 
naturaleza química de los mismos. Gonzaga y col. (2005) señala que los principales metabolitos presentes en este género son los flavonoides de tipo flavanona y flavona; y los diterpenos de núcleo kaurano, clerodanos y labdano. Las propiedades biológicas de estas especies se atribuyen a los flavonoides y los diterpenos.

Los ensayos cromatográficos son importantes para determinar el número de compuestos presentes $\mathrm{y}$ el sistema cromatográfico más adecuado para poder separarlos. Se utilizó mezclas de cloroformo y metanol; y el mejor sistema fue $\mathrm{CHl}_{3}: \mathrm{MeOH}(12: 1)$, obteniéndose hasta cinco fracciones bien definidas (Kuklinski, 2000). Los compuestos fueron caracterizados como flavonoides por su fluorescencia y sus pruebas características. En las tablas 2, 3 y 4 , se reportan las pruebas características de identificación para flavonoides con sus datos espectrales. En las tres especies se puede confirmar la presencia de flavonas y flavononas por las lecturas espectrales (Mabry y col, 1970). Gonzaga y col (2005) señalan que en Baccharis genistelloides se han identificado 10 flavonas, flavanona y 10 diterpenos de núcleo clerodano; mientras que en Baccharis salicifolia $15 \mathrm{fla}$ vonas, 7 flavanonas y 22 diterpenos, 17 labdanos, 3 clerodanos y 2 triterpenos; y en Baccharis glutinosa una flavanona.

En la figura 1, se reporta los resultados del ensayo de la actividad antiinflamatoria de las tres especies, en donde $B$. salicifolia y $B$. gensitelloides tienen una actividad superior a $B$. glutinosa, utilizando como agente inflamatorio a la PGE1. Esta acción valida el conocimiento popular por el cual B. salicifolia es ampliamente utilizado como agente antiinflamatorioa, analgésico y antirreumático y su actividad es semejante a los AINES, mientras que $B$. genistelloides también tiene esta acción, pero en menor grado que $B$. salicifolia. $B$. genistelloides también es utilizado como antiinflamatorio y para problemas hepáticos. B. glutinosa si bien no tiene una buena actividad antiinflamatoria, podría tener otras propiedades biológicas aún no conocidas. La actividad antiinflamatoria se debe a los compuestos químicos presentes, principalmente a los flavonoides y a los triterpenos, como se confirma con los ensayos fitoquímicos realizados. El análisis de varianza muestra que existen diferencias estadísticamente significativas $(p<0.05)$, pero en la prueba de comparaciones múltiples o Prueba de Tukey aparentemente las tres especies tienen el mismo comportamiento farmacológico.
En la figura 2, se muestra el ensayo de la actividad antioxidante de las tres especies, en donde $B$. salicifolia muestra tener una gran actividad antioxidante superiror a B. gensitelloides y B. glutinosa. El gran número de flavonoides presentes en esta especie justifica su gran actividad antioxidante, además se sabe que los flavonoides de núcleo flavona muestran una gran actividad secuestradora del DPPH (Villar del Fresno, 1999) (Bruneton, 2001). Hirano et al. (2001) evaluaron la actividad de 10 flavonoides sobre el DPPH, y que esta actividad dependía de la naturaleza química. La quercetina (un flavonol) mostró una mayor actividad secuestradora que la luteolina. Middleton y col (2000), han hecho una amplia revisión de las propiedades farmacológicas de los flavonoides hallando que la actividad antiinflamatoria y la actividad antioxidante, están intimamente relacionadas y dependen de la estructura química, siendo las flavonas y los flavonoles, los que tienen mayor actividad. El análisis de varianza factorial de este ensayo muestra que existe diferencias entre las especies ensayadas $(p<0.05)$ y en menor grado respecto a la concentración.

En la tabla 5, se observan los resultados obtenidos del efecto antimicrobiano de las tres especies de Baccharis, observándose que a la concentración mayor trabajada de los extractos al $1 \%$, B. salicifolia y $B$. genistelloides no presentaron actividad antibacteriana a las tres bacterias estudiadas, Shigella sonnei, Salmonella $s p$ y Staphylococcus aureus. Por el contrario B. glutinosa sí presentó actividad frente a Salmonella sp y Shigella sonnei, mas no a Staphylo. coccus aureus. Al respecto, Salmonella sp y Shigella sonnei son bacterias gram negativas cuya composición de su pared celular, que es básicamente lipídica es diferente a una gram positiva, como lo es Staphylococcus aureus, razón por la cual B. glutinosa contiene sustancias capaces de atravesar la pared celular de dichas bacterias.

En conclusión, de las tres especies estudiadas Baccharis salicifolia es ampliamente conocida por nuestra población como antiinflamatoria, antirreumática y analgésica, conocimiento popular que se valida con los ensayos químicos y farmacológicos realizados y en menor grado Baccharis genistelloides. Mientras que Baccharis glutinosa requiere estudios más profundos para revelar las propiedades medicinales que pueda poseer, a pesar que la población la confunde con Baccharis salicifolia. 


\section{AGRADECIMIENTOS}

El presente trabajo de investigación se desarrolló con auspicio de la Universidad Nacional de San Cristóbal de Huamanga. Agradecimientos a los profesores Mg. Jesús de la Cruz, Martha Romero y a la estudiante Carol Pillaca.

\section{REFERENCIAS BIBLIOGRÁFICAS}

1. Arones M. Estudio fitoquimico de Baccharis salicifolia y determinación de su actividad antiinflamatoria. Tesis-UNSCH. Ayacucho. 1998.

2. Brack A. Diccionario Enciclopédico de plantas útiles del Perú. Centro de Estudios Regionales Andinos Bartolomé de las Casas. Programa de las Naciones Unidas para el Desarrollo (PNUD). Cusco. 1999. p 556.

3. Bruneton J. Plantas Medicinales, Fitoquímica y Farmacognosia. Editorial Acribia. Zaragoza. 2001.

4. Cáceres A. Plantas Medicinales de Uso Medicinal en Guatemala. Universidad San Carlos. Guatemala. 1996.

5. Castro Y. Actividad citoprotectora de lactonas sesquiterpénicas aisladas de Baccharis genistelloides. Tesis-UNSCH. Ayacucho. 2000.

6. CYTED. Manual de Técnicas de Investigación. Programa Iberoamericano de Ciencia y Tecnología para el Desarrollo. 1995.

7. De la Cruz J y Romero M. Asteráceas en el Distrito de Huamanguilla. Inf. de Inv. .Fac. Cs. Bs.UNSCH.1997.

8. De la Cruz J y Romero M. Estudio Botánico y Taxonómico de las Asteraceas en el transecto Ayacucho - Niñobamba. Inf. Inv. Fac. Cs. Bs.- UNSCH. 1999.
9. Gonzaga L, Costa I y Geraldo M. Género Baccharis (Asteraceae): Aspectos Químicos, Económi$\cos$ y Biológicos. Quim. Nova, Vol. 28, No. 1, 8594,2005

10. Hirano R, Sasamoto W, Matsumoto A, Itakura $\mathrm{H}$, Igarashi $\mathrm{O}$ y Kondo $\mathrm{K}$. Antioxidant ability of various flavonoids against DPPH radicals and LDL oxidation. J Nutr Sci Vitaminol. (2001)47(5):357-62.

11. Kuklinski C. Farmacognosia. Estudio de las Drogas y Sustancias Medicamentosas. Ediciones Omega. Barcelona. 2000.

12. Lock de Ugaz O. Investigación Fitoquímica: Métodos en el Estudio de los Productos Naturales. Fondo Editorial de la Universidad Católica del Perú. Lima. 1994.

13. Mabry T, Markam K y Thomas M. The Systematic Identification of Flavonoids. Springer-Verlag. New Cork. 1970.

14. Middleton E, Kandaswami C, Theoharides T. The Effects of Plant Flavonoids on Mammalian Cells: Implications for Inflammation, Heart Disease, and Cancer. Pharmacol Rev. (2000) 52:673-751

15. Palacios J. Plantas Medicinales Nativas del Perú. Serie Ciencias. CONCYTEC. Lima. 1997.

16. Suárez S. Antioxidantes en recursos Fitoterapéuticos. Primer Curso Nacional. Centro de Investigación de Bioquímica y Nutrición. Facultad Medicina. UNMSM. 2006.

17. Tovar O. Plantas Medicinales del Valle del Mantaro. CONCYTEC. Lima. 2001.

18. Villar del Fresno M. Farmacognosia General. Editorial Síntesis. Madrid. 1999. 